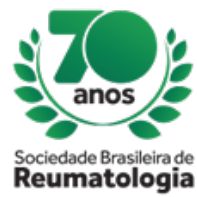

\title{
ULTRASSONOGRAPHIC AND METABOLIC ANALYSIS IN PSORIASIS AND PSORIASIS ARTHRITIS.
}

José Alexandre Mendonça (PUC Campinas, Campinas, SP, Brasil), Lucas Nogueira Pasani (PUC Campinas, Campinas, SP, Brasil), Mateus Basso Mimoto (PUC Campinas, Campinas, SP, Brasil), ELAINE CRISTINA FARIA ABRAHÃO MACHADO (PUC Campinas, Campinas, SP, Brasil), Ana Carolina Bazan (PUC

Campinas, Campinas, SP, Brasil), Igor Tadeu Garcia Ferreira (PUC Campinas, Campinas, SP, Brasil), Fernanda Bertucci Sanches (PUC Campinas, Campinas, SP, Brasil)

\section{BACKGROUND}

Psoriatic arthritis and psoriasis have a broad clinical spectrum, which involves inflammation of the tendon, enthesis, and alteration of the trilaminar pattern of the nails by ultrasonography. They are very associated with cardiovascular diseases and metabolic syndrome, being the main reasons involved in the mortality of these patients. Ultrasound was able to evaluate the inflammatory activity of these diseases and also to estimate cardiovascular risk by carotid study. Considering the greater predisposition of these patients, and the great cardiovascular impact. In this study, we analyzed the carotid, other metabolic variables and ultrasonographic findings typical of psoriasis and psoriasis, and evaluated for correlation [1,2].

Objectives: To detect and compare changes indicating inflammatory and atherosclerosis process in 2 groups of patients, using Doppler velocimetric indexes articular and quantitative media thickness software (QIMT) for the carotid artery.

\section{MATERIALS AND METHODS}

Thirty patients were evaluated: 15 in the psoriasis carrier group (134 nail beds; 30 carotid arteries); and 15 in the group with psoriatic arthritis (147 nail beds; 30 carotid arteries). "CASPAR" criteria were used to classify the patients. The ultrasound (US) was performed in all patients by a single rheumatologist using Esaote MyLab 50. Was collected laboratory tests for evaluation of disease activity and metabolic profile, later submitted to Spearman and Pearson correlation.

\section{RESULTS}

Table 1

A statistically significant difference was found in the NAPSI and variable E among the groups $p<0.05$ and $p=0.006$ respectivily.

Spearman and Pearson correlations between US variables per group, psoriasis, psoriatic arthritis were: PASIXSG $r=0,658, p=0,015$, VHSxPI varying $r$ from $-0,387$ to $-0,614$ and $p$ varying from 0,038 to 0,002 ; FExRI varying $r$ from 0,351 to 0,526 and $p$ varying from 0,034 to 0,002 , VHSxRI varying $r$ from $-0,329$ to $-0,620$ and $p$ varying from 0,04 to $0,001, S G x R I$ varying $r$ from 0,542 to 0,694 and $p$ varying from 0,001 to 0,001 . The prevalence of metabolic syndrome, were: $53,3 \%(8)$ in the psoriasis group, and $30 \%(5)$ in the arthritis group. 53,3\% (8) in the psoriasis group had QIMT-RF above the QIMT expected; in the arthritis group, $40 \%$.

\section{CONCLUSION}

The data of this study, obtained an association between the metabolic and inflammatory variables, it also shows data that point to a poorer metabolic profile and nail thickening in patients with psoriasis.

These patients should be regarded as high-risk cardiovascular patients. And the concomitant control of the inflammatory and metabolic aspects is mandatory in the conduction of these patients. 